\title{
Vinzolidine Sulfate
}

National Cancer Institute

\section{Source}

National Cancer Institute. Vinzolidine Sulfate. NCI Thesaurus. Code C87288.

The sulfate salt of vinzolidine, an orally active semisynthetic vinca alkaloid with potential antineoplastic activity. Like other vinca alkaloid compounds, vinzolidine binds to and stabilizes tubulin molecules, thereby interfering with microtubule assembly/disassembly dynamics. As a result, vinzolidine prevents mitotic spindle formation and leads to cell cycle arrest in metaphase. 